\title{
Modification of aftertaste with a menthol mouthwash reduces food wanting, liking, and ad libitum intake of potato crisps
}

Scott. C. Hutchings, Katy M. Horner, Victoria A. Dible, John M. V. Grigor, and Dolores O'Riordan.

This is the accepted manuscript $@$ 2016, Elsevier Licensed under the Creative Commons Attribution-NonCommercialNoDerivatives 4.0 International: http://creativecommons.org/licenses/bync-nd/4.0/

$((c))$ EY-No-ND

The published article is available from doi: 10.1016/j.appet.2016.09.022 
Modification of aftertaste with a menthol mouthwash reduces food wanting, liking, and ad libitum intake of potato crisps

Scott. C. Hutchings ${ }^{\mathrm{a}, *}$, Katy M. Horner ${ }^{\mathrm{b}}$, Victoria A. Dible ${ }^{\mathrm{b}}$, John M. V. Grigor ${ }^{\mathrm{c}}$, \& Dolores O’Riordan ${ }^{\mathrm{b}}$.

${ }^{a}$ Faculty of Veterinary and Agricultural Sciences, The University of Melbourne, Parkville, Victoria, Australia

${ }^{\mathrm{b}}$ Institute of Food and Health, School of Agriculture and Food Science, University College Dublin, Belfield, Dublin, Ireland

${ }^{\mathrm{c}}$ School of Science, Engineering \& Technology, Abertay University, Dundee, United Kingdom

\begin{abstract}
This research investigated the effect of modifying the aftertaste of potato crisps on (1) temporal sensory perception and (2) appetite using three mouthwash conditions (no mouthwash, a water mouthwash, and a menthol mouthwash). For the sensory study, 17 screened female subjects were trained on the Temporal Dominance of Sensations (TDS) methodology. Subjects undertook TDS to monitor all sensory attributes during the mastication of a $2 \mathrm{~g}$ crisp until swallowing (at $20 \mathrm{~s}$ ), then conducted the mouthwash, and then continued the TDS task to monitor aftertaste until 90s. For the appetite study, 36 subjects (18 male, 18 female) completed 100mm Visual Analogue Scales (VAS) for desire, liking, hunger, and thirst, followed by an ad libitum eating task. For the VAS scales testing, subjects chewed and swallowed a $2 \mathrm{~g}$ crisp, and then immediately conducted the mouthwash before completing the VAS scales. For the ad libitum task, subjects were given 12 minutes to consume as many crisps as they desired on a plate (up to 50g). Every three minutes they were required to conduct a mouthwash. TDS results showed that in comparison with no mouthwash, the water mouthwash significantly reduced aftertaste attributes such as savoury, salty, and fatty mouthcoating, and the menthol mouthwash significantly increased aftertaste attributes of cooling, minty, and tingly. The water mouthwash did not influence desire and liking of crisps, or hunger and thirst. The water mouthwash did not influence $a d$ libitum intake of the crisps over a 12 minute period. The menthol mouthwash significantly reduced desire and liking of the crisps, as well as hunger and thirst. Furthermore, the menthol mouthwash significantly reduced ad libitum crisp intake by $29 \%$ over the 12 minute period.
\end{abstract}

Keywords: Food intake; sensory cues; liking; wanting; temporal dominance of sensations; aftertaste 


\section{Highlights:}

- $\quad$ Crisp aftertaste was assessed by 3 mouthwash conditions: no wash, water, menthol.

- TDS was used to monitor changes in crisp aftertaste with each condition.

- Water mouthwash did not influence appetite or intake of potato crisps.

- Menthol mouthwash reduced appetite and intake of potato crisps. 


\section{Introduction}

Excessive snacking of energy dense foods plays a significant role in the growth of the obesity epidemic (Forslund et al., 2005). Consumers, nutritionists, and food scientists are all seeking novel approaches to reduce the quantity that such products are consumed in. One approach to reduce energy intake is through the modification of sensory properties of foods (McCrickerd \& Forde, 2015). Increasing orosensory exposure has been shown to increase satiety through the reduction of sip size in beverages (Weijzen et al., 2009), and the texture of solid foods has been shown to increase satiety as harder foods require greater oral processing effort than soft foods (Forde et al., 2013 a \& b). An increase of retronasal aroma release has also been shown to increase satiety (Ruijschopa et al., 2008), and in some cases can influence food intake Ramaekers et al. (2014a).

Promising research is also emerging by changing the type of aroma, where the presence of incongruent odours was found to decrease appetite (Ramaekers et al., 2014 b \&c). Ramaekers et al. (2014 b \& c) showed that where an odour differed greatly from the food by which participants were questioned, appetite decreased. Savoury aromas decreased the appetite for sweet foods, and sweet aromas decreased the appetite for savoury foods. Non-food odours also decreased appetite. This research somewhat challenges conventional thinking in regards to sensory specific satiety (SSS) (Rolls et al., 1981; Rolls $\&$ Rolls, 1997), but offers significant potential for appetite and food intake control. Furthermore, the presence of a particular odour has been shown to promote or 'prime' the selection of foods with associated sensory properties. Gaillet et al. (2013) and Gaillet et al. (2014) showed that priming subjects with a melon scent caused an increase in the likelihood to select vegetables in a starter from a menu, and priming subjects with a pear scent increased the likelihood to choose desserts with fruit.

It is hypothesised that changing sensory perception via a mouthwash after swallowing could modify aftertaste in a way that reduced desire for unhealthy snacks. It is possible this could be achieved by removing the aftertaste generated by unhealthy snacks with a water mouthwash, or by the addition of a mouthwash with an incongruent sensory profile to the food being consumed. This could lead to the development of practical strategies for consumers to curb their snacking behaviour, such as intervening during a snacking occasion by consuming an everyday product that has a contrasting taste profile shown to suppress appetite for high fat snack. In years to come, it may also be possible develop the technology where incongruent tastes can be released from within unhealthy products after a specific period of time into an eating event, to prevent overconsumption.

One compound which may provide a suitable sensory profile to reduce the intake of such snacks is menthol. For a salty, high fat snack such as crisps, menthol offers a complete sensory contrast, through minty, cooling and tingling sensations (Eccles, 1994). Its sensation is linked to a number of everyday products that are not associated with the intake of food, such as toothpaste, chewing gum, and dental mouthwash. 
To assess the modification of aftertaste using a mouthwash, a dynamic sensory method is needed to monitor changes in perception with time. The Temporal Dominance of Sensations (TDS) technique is a relatively new sensory methodology (Labbe et al., 2009) used to assess changes in multiple sensory attributes with time: throughout the process of mastication (Hutchings et al., 2014a) and after swallowing ( $\mathrm{Ng}$ et al., 2012). While some earlier techniques have allowed several attributes to be monitored at the same time (Duizer et al., 1997; Green \& Hayes, 2003), TDS allows numerous attributes (typically 8-12) to be monitored at the same time, where subjects are required to select the most dominant sensory attribute, and change their selection if and when they feel the most dominant attribute changes. The training required to assess 8-12 attributes with TDS is also very small in comparison to the large amounts of time that would be required for traditional time intensity methods with 8-12 attributes (Pineau et al., 2009). Consequently, the TDS technique has the potential to effectively assess the sensory profile of the mastication of a solid food product immediately followed by the aftertaste of a mouthwash within the same chewing, swallowing, and mouthwash sequence.

The aim of the study was to:

(1) Assess the influence of a water mouthwash and menthol mouthwash on the aftertaste of potato crisps using the Temporal Dominance of Sensations methodology (in comparison with a 'no mouthwash' control).

(2) Assess the effect of a water mouthwash and menthol mouthwash on food liking, desire, hunger, thirst, and ad libitum food intake of potato crisps (in comparison with a 'no mouthwash' control). 


\section{Methodology}

\subsection{Overall design}

This research was approved by the University College Dublin Human Ethics Committee (Application LS-E-15-90) and all subjects provided informed consent prior to participation. The research was broken into two studies. The first study sought to establish the sensory implications of each mouthwash treatment on the aftertaste of potato crisps using the Temporal Dominance of Sensations (TDS) methodology. The second study sought to understand the influence the mouthwashes were having on appetite for potato crisps using visual analogue scales (VAS) and an ad libitum eating task. The two studies were conducted separately with different participants. This was designed to ensure naïve consumers could be tested for appetite using VAS scales, without the confounding effect of using a complex sensory method like TDS at the same time. It also allowed for appetite to be measured immediately after the mouthwash was applied. The essence of the work was that in both studies subjects would masticate and then swallow a $2 \mathrm{~g}$ crisp, before immediately applying a mouthwash to influence aftertaste. Mastication time (from the commencement of chewing until swallowing) was controlled at 20 s for the sensory study. For the appetite study subjects were instructed to chew and swallow in a manner that felt natural and comfortable for them. The methodology used for the sensory study is summarised in Fig. 1, and the methodology used for the appetite study is summarised in Fig. 2. 


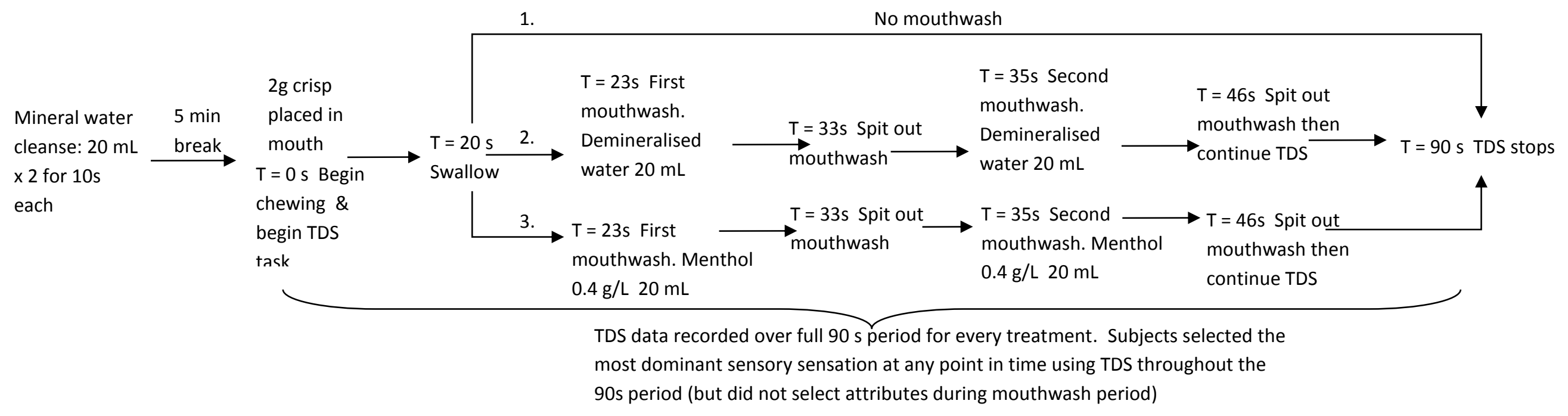

Fig. 1: A summary of the methodology used for the Temporal Dominance of Sensations (TDS) (sensory) study. Throughout mastication and swallowing of the crisp (0-23s), subjects continuously selected the most dominant sensory attribute they perceived from a list of attributes using a computer (with TDS sensory software) and mouse, changing their sensory attribute selection at any time if desired. From $23 \mathrm{~s}-46 \mathrm{~s}$ subjects refrained from attribute selection as they were undertaking the mouthwash. From 46s-90s subjects recommenced selecting the most dominant sensory attributes for the aftertaste experience, again changing their attribute selection at any time if desired. 
$3 \mathrm{hr}$ fast after breakfast

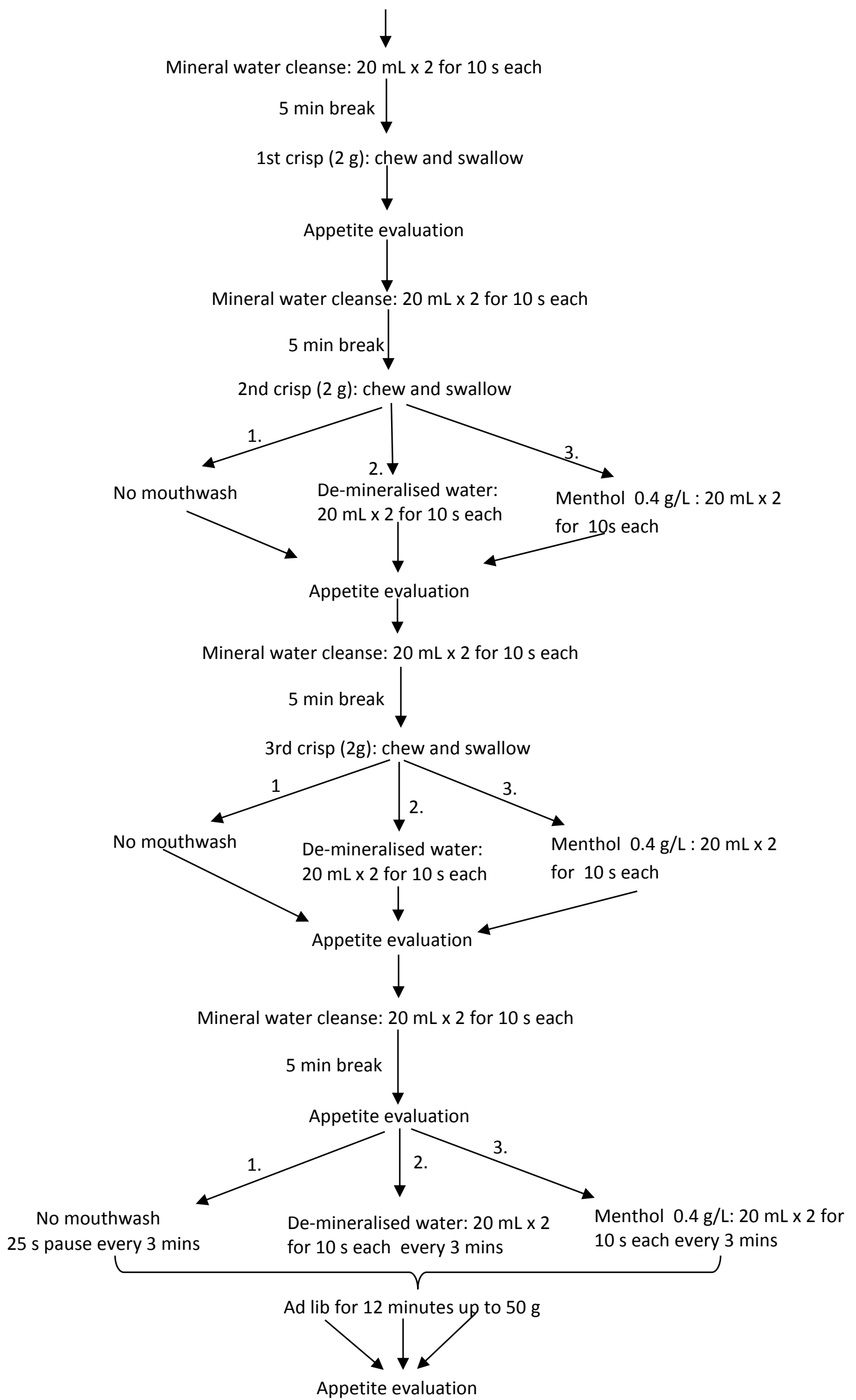

Fig. 2: A summary of the methodology used for the appetite study. 


\subsection{Treatments}

The crisps used for both studies were ready salted crisps (Pringles ${ }^{\circledR}$, Kelloggs, Poland) (2161 kJ per 100g). Three mouthwash treatments were used in both studies: 1 - no mouthwash, 2 - a water mouthwash using demineralised water (VWR Chemicals, Dublin, Ireland), 3 - a mouthwash of menthol at $0.4 \mathrm{~g} / \mathrm{L}$. Both mouthwash treatments involved subjects washing $20 \mathrm{~mL}$ of the mouthwash for $10 \mathrm{~s}$, expectorating the entire sample, and then repeating with another $20 \mathrm{~mL}$ of mouthwash (fresh mouthwash, not the same sample) for $10 \mathrm{~s}$ and expectorating the entire sample. The mouthwash was conducted twice to ensure a thorough wash of the mouth and/or application of menthol taste. The preparation of a $0.4 \mathrm{~g} / \mathrm{L}$ menthol solution involved dissolving $0.2 \mathrm{~g} \mathrm{~L}-\mathrm{Menthol}$ crystals (99\%, Sigma Aldrich, Steinheim, Germany) in $5 \mathrm{~mL}$ of food grade ethanol (Sedacol, 96\%, Selby, UK). Demineralised water (500 mL) (VWR Chemicals, Dublin, Ireland) was then heated to $60{ }^{\circ} \mathrm{C}$, and the ethanol-menthol mixture was then added to the water, and mixed in a sealed container. The final menthol solution had an ethanol content of $1 \% \mathrm{v} / \mathrm{v}$. All solutions and crisps were served at $20^{\circ} \mathrm{C}$ in isolated sensory booths of standard dimensions, with a room temperature of $20^{\circ} \mathrm{C}$ and standard white light.

\subsection{Palate cleansing procedure}

Throughout the sensory and appetite studies (prior to each crisp (eg a chew/swallow/mouthwash sequence) and prior to the ad libitum task), subjects were asked to cleanse their mouth and then wait five minutes. This involved washing the mouth with $20 \mathrm{~mL}$ of mineral water (Ballygowan ${ }^{\circledR}$, Britvic Ireland Ltd., Dublin, Ireland) for 10s, expectorating, and then washing with another $20 \mathrm{~mL}$ of mineral water for 10s, expectorating, and then lightly drying surface of the tongue with a piece paper towel to remove excess moisture (the paper towel was then immediately disposed of), before waiting five minutes. This approach was designed to minimise any lingering flavours from the crisps or mouthwashes, and to allow time for the moisture content in the mouth to equilibrate. This procedure (including the 5 minute break) was even enforced prior to the first crisp to allow time for the mouth to equilibrate in this way. It also maintained a consistent protocol with all other stages in the study.

\subsection{Sensory study}

\subsubsection{Selection of subjects for TDS study}

Eighteen female subjects aged 20-23 (average age: 22 years) were recruited within the Food Science and Agriculture department at University College Dublin. Subjects all had some knowledge of sensory science but were not trained panellists. Subjects did not suffer from any health conditions that 
influenced their ability to taste or chew. Subjects were screened through an identification test, where they were required to correctly identify the tastes of unknown solutions: sweet (sucrose $10 \mathrm{~g} / \mathrm{L}$, Sigma Aldrich ${ }^{\circledR}$, Steinheim, Germany), salty (sodium chloride 2 g/L, Sigma Aldrich ${ }^{\circledR}$ ), and savoury (monosodium glutamate, $0.6 \mathrm{~g} / \mathrm{L}$, Sigma Aldrich ${ }^{\circledR}$ ), and required to correctly identify unknown odours of vanilla (Vanilla essence, Goodall's, Dublin, Ireland), almond (Almond essence, Goodall's), and mint (peppermint essence, Goodall's). De-mineralised water was used to prepare solutions. To be eligible to take part in this study, subjects needed to score 5 out of 6 or greater. Answers near to the taste or flavour (eg meaty or umami for savoury) were also accepted as correct.

\subsubsection{Attribute generation and reference selection}

To generate attributes subjects were asked to consume a crisp and then undertake the mouthwash procedures. They recorded sensory terms they experienced during chewing, and also sensory terms experienced after swallowing/after each mouthwash. Through consensus the following twelve attributes were selected for the TDS study: salty, savoury, potato-like, crispy/crunchy, brittle/sharp, mushy, watery, fatty mouthcoating, tingly, cooling, mint, and no taste. Appropriate references were then selected for each attribute by the researchers, as well as a definition for each attribute. This is summarised in Table 1.

Table 1: Sensory attributes, definitions, and references used for the TDS study.

\begin{tabular}{|c|c|c|}
\hline Attribute & Description/Definition & Reference Food \\
\hline Salty & $\begin{array}{l}\text { Basic taste associated with } \\
\text { sodium chloride/table salt. }\end{array}$ & $\begin{array}{l}\mathrm{NaCl}(2 \mathrm{~g} / \mathrm{L}) \text {. Sigma Aldrich, } \\
\text { Germany. }\end{array}$ \\
\hline Savoury & $\begin{array}{l}\text { A brothy, rich or meaty } \\
\text { sensation. }\end{array}$ & $\begin{array}{l}\text { Savoury solution (MSG) (0.6 } \\
\text { g/L). Alamgeer, Pakistan. }\end{array}$ \\
\hline Crispy/ Crunchy & $\begin{array}{l}\text { Sensation of a loud sound } \\
\text { under the force of the teeth } \\
\text { during biting and chewing. }\end{array}$ & Cornflakes. Kelloggs, UK. \\
\hline Brittle/ Sharp & $\begin{array}{l}\text { A tendency to crack, fracture or } \\
\text { shatter without substantial force } \\
\text { by the teeth. }\end{array}$ & $\begin{array}{l}\text { Poppadoms. Sharwoods, } \\
\text { Premier Foods, UK. }\end{array}$ \\
\hline Mushy & A soft and pulpy texture. & Ripe Banana. Fyffes, Ireland. \\
\hline Potato-like & Like the flavour of potato. & $\begin{array}{l}\text { Boiled Potato. Rooster } \\
\text { potatoes. Keoghs, Ireland. }\end{array}$ \\
\hline Fatty mouthcoating & $\begin{array}{l}\text { Degree of residual oiliness left } \\
\text { on the oral cavity after } \\
\text { swallowing sample. }\end{array}$ & $\begin{array}{l}\text { Coconut oil. Cocowel, } \\
\text { Thailand. }\end{array}$ \\
\hline Watery & Feeling of total wetness. & $\begin{array}{l}\text { Mineral Water, Ballygowan, } \\
\text { Ireland. }\end{array}$ \\
\hline
\end{tabular}




\begin{tabular}{|l|l|l|}
\hline Minty & The taste of peppermint. & $\begin{array}{l}\text { Peppermint lolly. Silvermints, } \\
\text { Valeo Foods, Ireland. }\end{array}$ \\
\hline Cooling & $\begin{array}{l}\text { Cooling sensation on all } \\
\text { surfaces of oral cavity. }\end{array}$ & $\begin{array}{l}\text { Airwaves chewing gum. } \\
\text { Wrigleys, UK. }\end{array}$ \\
\hline Tingly & $\begin{array}{l}\text { A feeling of increased sensation } \\
\text { in the mouth. }\end{array}$ & $\begin{array}{l}\text { Listerine mouthwash. Johnson } \\
\text { \& Johnson, UK. }\end{array}$ \\
\hline No taste & Lacking flavour. & No reference \\
\hline
\end{tabular}

\subsubsection{Training on the TDS technique and the use of mouthwashes}

Each subject underwent a training exercise of approximately 90 minutes. Subjects were told about the TDS technique, and then trained on the definition of each attribute using the reference products. They were then taken into isolated sensory booths to be trained on the use of TDS using FIZZ sensory software (Biosystems, Couternon, France) with a computer. During the training session they conducted 4 separate assessments of 'sour cream \& onion' flavoured crisps (one $2 \mathrm{~g}$ crisp per assessment) (Pringles ®, Kelloggs, Poland). This flavour differed from the actual experiment so that training did not affect sensory results. They were also trained on the mouthwash procedure. Subjects were under strict instructions not to use TDS during the mouthwash period. By accompanying subjects in the sensory booth during training, the researchers could check the mouthwash procedure was undertaken correctly and that TDS was not being conducted at the same time. Using standard TDS procedure, subjects were told to select the most dominant sensation (attribute) they perceived at any point in time, and if that dominant sensation changed at any point in time they can change their selection. They could change the selection of the dominant sensation as often as they liked, but could only select one attribute at a time.

\subsubsection{Sensory session (using TDS)}

The TDS session was undertaken by each subject using FIZZ sensory software on a computer inside the sensory booth. Each subject took part in three sensory sessions (one for each mouthwash treatment: no mouthwash, water mouthwash, and menthol mouthwash). Mouthwash treatments were conducted in separate sessions, rather than in the same session, to simulate similar conditions to the appetite study. This allowed the researchers to assess if the prior mouthwash condition influenced sensory perception (during mastication) before the subsequent mouthwash (particularly important for menthol).

The order of sessions and the order of attributes were counterbalanced using Latin-square designs. In each session, four replicate sequences were conducted. Subjects placed the single crisp into their mouth (2g), clicked start, and then began to chew while selecting the most dominant attribute. Alerts appeared 
beside the list of TDS attributes to guide the subjects. At 20 seconds they were alerted to swallow the crisp. At 23 s they were alerted to take the first mouthwash, and then at $33 \mathrm{~s}$ to spit it out. At $36 \mathrm{~s}$ seconds they were alerted to take the second mouthwash, and then at $46 \mathrm{~s}$ instructed to spit it out. They were then alerted to continue with the selection of TDS attributes until at 90s, where the TDS task stopped. Between each crisp, and before the first crisp, the 5 minute palate cleansing procedure was applied (section 2.3). The timings were designed to mimic the timings used by subjects in the appetite study that followed. Each session lasted approximately 30 minutes in total.

\subsubsection{Data analysis for TDS study}

TDS data from every subject and replicate was combined to form a standard TDS curve according to previous literature (Pineau et al. 2009; Pineau \& Schlich, 2014). As the period of data collection was controlled (90s for every subject), unstandardized TDS curves were constructed. The procedure involves calculating the average percentage selection for every sensory attribute across the data from all subjects and replicates, at each time point. Data is plotted and curves formed using a Bezier smoothing function in FIZZ sensory software.

Subjects did not undertake TDS during the mouthwash period (23s-46s), however data collection continued during this period as the FIZZ software could not be temporarily switched off (the last attribute selected as dominant prior to the mouthwash continued to be selected). Therefore, none of the data collected during this period is analysed (as it is meaningless data), and it is blocked out on the TDS curves $(23 \mathrm{~s}-46 \mathrm{~s})$. 


\subsection{Appetite study}

\subsubsection{Subjects}

Test subjects were recruited from around campus at University College Dublin. A total of thirty-six subjects participated, eighteen males and eighteen females, aged 18 - 32 years (average age: 24 years). To ensure subjects had no expectation about the study, none of the subjects who took part in the sensory study took part in the appetite study. Furthermore none of the subjects had any prior knowledge of the study or its objectives. Subjects met strict screening criteria: they were all regular consumers of potato crisps (at least once per month), were not on a diet, did not suffer from any health conditions that influenced their appetite, and did not suffer from any health conditions that influenced their ability to taste or chew food. Subjects had a mean (SD) body mass index of $23.2( \pm 2.2) \mathrm{kg} / \mathrm{m}^{2}$, waist circumference $77.4( \pm 7.8) \mathrm{cm}$, TFEQ dietary restraint score $8.2( \pm 3.8)$, disinhibition score $5.6( \pm 2.9)$ and hunger score $6.9( \pm 2.7)$. All subjects completed the three test sessions.

\subsubsection{Design}

Every subject completed three test sessions (no mouthwash, water mouthwash, and menthol mouthwash) on separate days, where the order was counterbalanced according to a Latin square design. No replicate sessions were conducted. Sessions were conducted a minimum of four and maximum of seven days apart. On each test day, subjects were instructed to have their typical breakfast at the time they normally have breakfast, and then attend the laboratory three hours later. Subjects were instructed to have the same breakfast on every day of the test and prior food intake was checked by a study investigator on each test day. Subjects were not allowed to eat any food or drink any beverages (with the exception of water) and were instructed to minimise exercise in the three hour period between breakfast and the beginning of the study. The three test sessions were identical except for the manipulation of aftertaste. All sessions lasted approximately 45 minutes.

\subsubsection{Visual Analogue Scales (Ratings) Protocol}

To establish baseline measurements without a mouthwash, a single crisp (2g) was first served. Each subject was asked to chew and swallow the entire crisp at once (in a natural manner). No mouthwash was given in the case of this first crisp for every session. Once the crisp was swallowed, subjects were then required to immediately answer the following questions using a continuous $100 \mathrm{~mm}$ VAS scale (anchored 'not at all' on the left anchor, and 'very' on the right anchor). 1: Please rate the pleasantness of the crisps you just swallowed, 2: Take a look at the crisps beside you, how strong is your desire to eat (taste, chew and swallow) more crisps right now?, 3: How hungry do you feel right now?, 4: How 
thirsty do you feel right now ?. Questions were developed according to guidelines by Rogers \& Hardman (2015). A plate containing 50g of the crisps was placed beside each subject for stimulation during the questions (they were not allowed to consume from this plate until the ad libitum stage of the session.

The second crisp was then served and consumed in the same manner (2g). Immediately after swallowing this second crisp, subjects where then asked to either have 1: no mouthwash, 2: the water mouthwash (10s x 2), or 3: the menthol mouthwash (10s x 2). They were then asked to immediately answer the same questions using the VAS scales. This process allowed all appetite ratings for the $2^{\text {nd }}$ crisp to occur only as a result of the aftertaste modification by mouthwash after the $2^{\text {nd }}$ crisp is swallowed (there was therefore no possible influence of carryover for the second crisp).

Then a third crisp was served followed by the same procedure, either 1: no mouthwash, 2: the water mouthwash (10s x 2), or 3: the menthol mouthwash (10s x 2) and then the VAS scales questions, to obtain data from a subsequent crisp. This allowed for an additional reading to compare with the $2^{\text {nd }}$ crisp, and the 5 minute palate cleansing procedure reduced any sensory based carry over effects from the $2^{\text {nd }}$ to $3^{\text {rd }}$ crisp (5 minute palate cleansing procedure was applied before every crisp/swallow/mouthwash event throughout the appetite study (section 2.3)).

\subsubsection{Ad Libitum intake task}

The ad libitum task was conducted at the end of each VAS test session. The 5 minute palate cleansing procedure (section 2.3) was again applied after the VAS test session, before the ad libitum task began. To account for any changes in appetite during the session, identical VAS questions were conducted before and after the ad libitum task. For the ad libitum task, each subject remained in the same closed sensory cubicle and was instructed to eat as many crisps as they liked from a $50 \mathrm{~g}$ serving. The task lasted 12 minutes in duration. At $\mathrm{t}=0 \mathrm{~min}, \mathrm{t}=3 \mathrm{~min}, \mathrm{t}=6 \mathrm{~min}$, and $\mathrm{t}=9 \mathrm{~min}$, subjects were required to either 1 = pause for $25 \mathrm{~s}, 2=$ conduct the water mouthwash $(10 \mathrm{~s} \times 2)$, or $3=$ conduct the menthol mouthwash (10s x 2).

\subsubsection{Additional data collection}

At the completion of each subject's final session, they were asked to complete the Three Factor Eating Questionnaire to assess any possible influence of dietary restraint, disinhibition or hunger traits on findings. Height, weight, and waist circumference were also recorded. 


\subsubsection{Data analysis for appetite study}

Statistical analysis was undertaken using IBM SPSS (version 20.0 for Windows) (IBM Corp., USA). Data from the VAS scales (ratings for pleasantness, desire, hunger, and thirst), and from the ad libitum task, was analysed using the Friedman test, as not all sections of the data were normally distributed according to the Shapiro-Wilk test for normality. To account for any differences in baseline readings from session to session for each subject, all subsequent statistical analyses calculations for visual analogue scale ratings dealt with data where the baseline measurement (1st crisp) reading was subtracted. 


\section{Results}

\subsection{Sensory study}

Changes in the temporal sensory perception of the crisps during mastication and after swallowing using the Temporal Dominance of Sensations (TDS) methodology is shown in Fig. 3. A similar trend in dominating attributes can be seen for all conditions during mastication of the crisps until swallowing (no mouthwash (1), water mouthwash (2), and menthol mouthwash (3)). During the early stages of mastication (0-10s), crispy/crunchy, brittle/sharp, and salty are selected at high dominance rates above the level of significance. After approximately 10 s the rate of selection of the crispy/crunchy and brittle/sharp attributes decline, however the salty attribute continues to be selected at a high rate above the level of significance throughout the mastication period. The selection of the savoury attribute also becomes prominent after approximately $10 \mathrm{~s}$, and remains selected at a rate above the level of significance until the point of swallow (at 20s). After approximately 12-13s the attributes mushy and potato-like begin to emerge as highly selected attributes above the level of significance.

Large differences in the TDS profiles emerge after the swallowing point as a result of the mouthwash treatments (Fig. 3). For the no mouthwash treatment (1), the rate of selection of the mushy and potatolike sensations reduces after swallowing. However, the savoury and salty attributes continue to be selected at a high dominance rates above the level of significance until the end of data collection at 90s. After approximately $25 \mathrm{~s}$, the fatty mouthcoating attribute becomes selected at a high dominance rate above the level of significance, and continues to be highly selected until 90s. After approximately 55 s the no taste attribute becomes selected above the level of significance, and its rate of selection continues to rise to just above $50 \%$ by 90 s.

For the water mouthwash treatment (2), the rate of selection of the salty and savoury attributes is greatly reduced in comparison with the no mouthwash treatment (1), falling well below the level of significance at $65 \mathrm{~s}$. Furthermore, the selection rate of the fatty mouthcoating attribute disappears almost entirely as a result of the water mouthwash. The selection of the watery attribute is well above the level of significance after the water mouthwash until approximately $80 \mathrm{~s}$. The water mouthwash also causes a large increase in the selection rate of the no taste attribute in comparison with the no mouthwash condition, as it rises rapidly after the water mouthwash, reaching over $80 \%$ by 90 s.

The application of the menthol mouthwash (3) greatly changes the aftertaste profile in comparison with the both other treatments $(1 \& 2)$. After the menthol mouthwash the selection of savoury, salty, and fatty mouthcoating attributes cease. The watery and no taste attributes also decline well below the chance level. The selection rate of the cooling and minty attributes rises rapidly, well above the level of significance throughout period after the mouthwash. The selection of the tingly attribute also emerges above the level of significance for most of the period after the mouthwash. 
It should be noted that the TDS curve does not start from the origin after the mouthwash. The dominant attribute at $23 \mathrm{~s}$ could not be unselected during the mouthwash period (and one attribute is always selected as dominant once the first attribute was selected according to the software). Consequently, the dominant attribute at 23 s could only be unselected (via selecting a new attribute) after the mouthwash (after 46s). Before the mouthwash the TDS curve starts from the origin as once the start button is clicked ( $\mathrm{t}=0 \mathrm{~s}$ ), data collection begins (and hence dominance rate calculations begin), but subjects typically take several seconds to make their first attribute selection. 

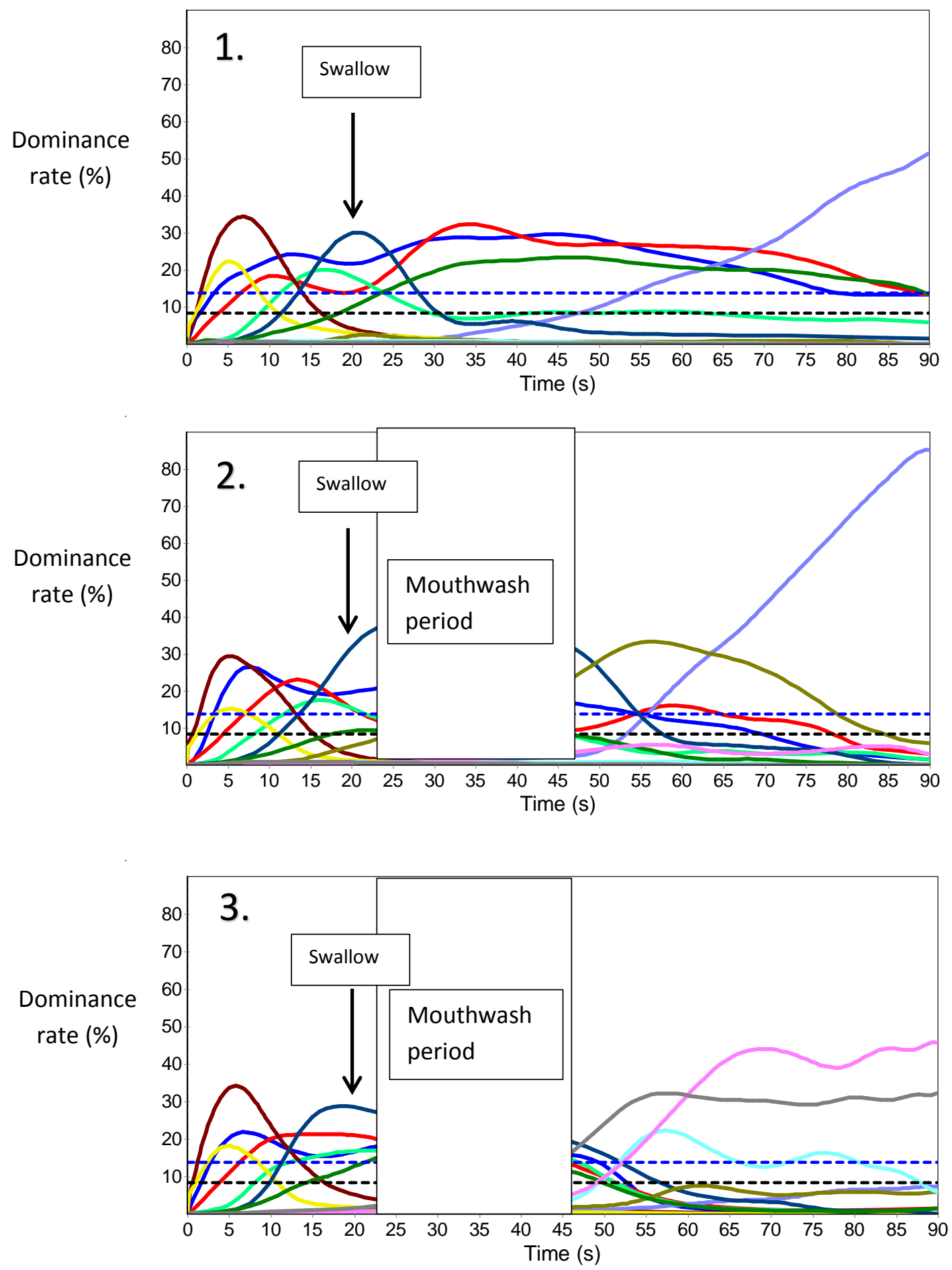

\footnotetext{
Attribute Salty

Attribute Savoury

Attribute Potato-like

Attribute Crispy/Crunchy

Attribute No taste

Attribute Brittle/Sharp

Attribute Mushy

Attribute Watery
}

Attribute Cooling

Attribute Minty

-... Chance Level

-・・Level of Significance (5\%) 
Fig. 3: Temporal Dominance of Sensations (TDS) curves showing the change in sensory perception during mastication and after swallowing over a 90s period. $1=$ no mouthwash, $2=$ water mouthwash, $3=$ menthol mouthwash. The curves describe how the percentage of selections (dominance rate \%) of the most dominant sensory attribute changes as the crisp is masticated, and after the mouthwash is applied. Dominance rate (\%) is calculated for all 12 attributes across all subjects and replicates at every time point (from 0s to 90s).

Subjects can only select one attribute at any point in time, but can change their choice as often as they like, and can return to the same attribute as often as desired. When an attribute is selected, a subject can stay on that attribute for as long as they desire. 


\subsection{Appetite study}

\subsubsection{Desire to eat more crisps}

VAS ratings for desire to eat more crisps during each test session are shown in Fig. 4. No significant difference was found in desire after the baseline first crisp (where none of the mouthwash treatments were applied) between mouthwash sessions according to the Friedman's test $\chi^{2}(2, N=36)=4.158, p>$ 0.05. A significant difference in desire for more crisps was found after the second crisp between mouthwash treatments according to the Friedman's test $\chi^{2}(2, N=36)=28.128, p<0.0005$. A significant difference in desire for more crisps was also found for the third crisp between mouthwash treatments according to the Friedman's test $\chi^{2}(2, \mathrm{~N}=36)=31.535, \mathrm{p}<0.0005$. Post hoc results are summarised in Fig. 4, showing desire after the second crisp and third crisp was significantly lower following the menthol mouthwash than the water mouthwash and no mouthwash treatments.

\subsubsection{Hunger}

VAS ratings for hunger during each test session are also shown in Fig. 4. There was no significant difference in hunger after the baseline first crisp (where no mouthwash treatments were applied) between mouthwash sessions according to the Friedman's test $\chi^{2}(2, N=36)=2.440, p>0.05$. A significant difference in perceived hunger was found after the $2^{\text {nd }}$ crisp between mouthwash treatments according to the Friedman's test $\chi 2(2, \mathrm{~N}=36)=17.720, \mathrm{p}<0.0005$. Results also show a significant difference in perceived hunger after the third crisp between mouthwash treatments according to the Friedman's test $\chi^{2}(2, \mathrm{~N}=36)=13.690, \mathrm{p}<0.005$. Fig. 4 shows the post hoc results. Hunger was significantly lower following the menthol mouthwash treatments after the second and third crisps.

\subsubsection{Pleasantness}

VAS ratings for pleasantness during each test session are shown in Fig. 4. There was no significant difference in perceived pleasantness of the crisps after the baseline first crisp (where no mouthwash treatments were applied) between mouthwash sessions according to the Friedman's test $\chi^{2}(2, N=36)=$ $1.282, p>0.05$. However, there was a significant difference in perceived pleasantness of the second crisp between different mouthwash treatments (mouthwashes applied after the second crisp was swallowed - the crisps were identical) $\chi^{2}(2, \mathrm{~N}=36)=6.606, \mathrm{p}<0.05$. There was also a significant difference in the perceived crisp pleasantness between mouthwash treatments for the third crisp (again mouthwash applied after third crisp was swallowed - the crisp was identical $) \chi^{2}(2, \mathrm{~N}=36)=10.746, \mathrm{p}$ 
$<0.05$. Post hoc results (Fig. 4) did not identify any pairwise differences in pleasantness after the second crisp, however the menthol mouthwash was shown to significantly reduce ratings by the third crisp.

\subsubsection{Thirst}

VAS ratings for thirst during each test session are also shown in Fig. 4. No significant difference was found in thirst after the baseline first crisp (where no mouthwash treatments were applied) between mouthwash sessions according to the Friedman's test $\chi^{2}(2, \mathrm{~N}=36)=0.884, \mathrm{p}>0.05$, or after the $2^{\text {nd }}$ crisp between mouthwash treatments according to the Friedman's test $\chi^{2}(2, \mathrm{~N}=36)=4.676, \mathrm{p}>0.05$. However results showed a significant difference after the $3^{\text {rd }}$ crisp between mouthwash treatments according to the Friedmans test $\chi^{2}(2, \mathrm{~N}=36)=7.627, \mathrm{p}<0.05$. For the third crisp, post hoc results (Fig. 4) show the menthol mouthwash significantly reduced thirst ratings.

\subsubsection{VAS ratings for ad libitum task}

The ad libitum exercise immediately followed the VAS scales tasks. While no there was no difference in VAS scale results for desire, hunger, pleasantness, or thirst when the baseline crisp ( $1^{\text {st }}$ crisp) was consumed, and an identical quantity of crisps was consumed during the VAS stage with identical timings, subjects entered the ad libitum task with different appetite due to the different mouthwash conditions at the VAS stage ( 5 minutes after completing the $3^{\text {rd }}$ crisp/mouthwash stage followed by a cleanse with mineral water). Before the ad libitum task there was a significant difference between mouthwash treatments in desire $\chi^{2}(2, \mathrm{~N}=36)=19.180, \mathrm{p}<0.0005$, hunger $\chi^{2}(2, \mathrm{~N}=36)=12.743, \mathrm{p}<$ 0.005 , pleasantness of the most recent crisp $\chi^{2}(2, \mathrm{~N}=36)=16.355, \mathrm{p}<0.005$, and thirst $\chi^{2}(2, \mathrm{~N}=36)$ $=10.652, \mathrm{p}<0.005$. After the ad libitum task no significant difference was observed between mouthwash treatments for desire $\chi^{2}(2, \mathrm{~N}=31)=1.918, \mathrm{p}>0.05$ or hunger $\chi^{2}(2, \mathrm{~N}=31)=0.443, \mathrm{p}>$ 0.05. However, there was a significant difference between mouthwash treatments in the pleasantness of the most recent crisp $\chi^{2}(2, \mathrm{~N}=31)=9.117, \mathrm{p}<0.05$ and thirst $\chi^{2}(2, \mathrm{~N}=31)=13.504, \mathrm{p}<0.005$. Post hoc results are summarised in Fig. 4, showing that desire, hunger, pleasantness (of the most recent crisp), and thirst was significantly lower for the menthol mouthwash session prior to the ad libitum task. Pleasantness (of the most recent crisp) and thirst remained significantly lower for the menthol mouthwash session after the ad libitum task. Fig. 4 also demonstrates that for all conditions hunger and desire decreased during the ad libitum task, whereas thirst increased and pleasantness remained steady. 


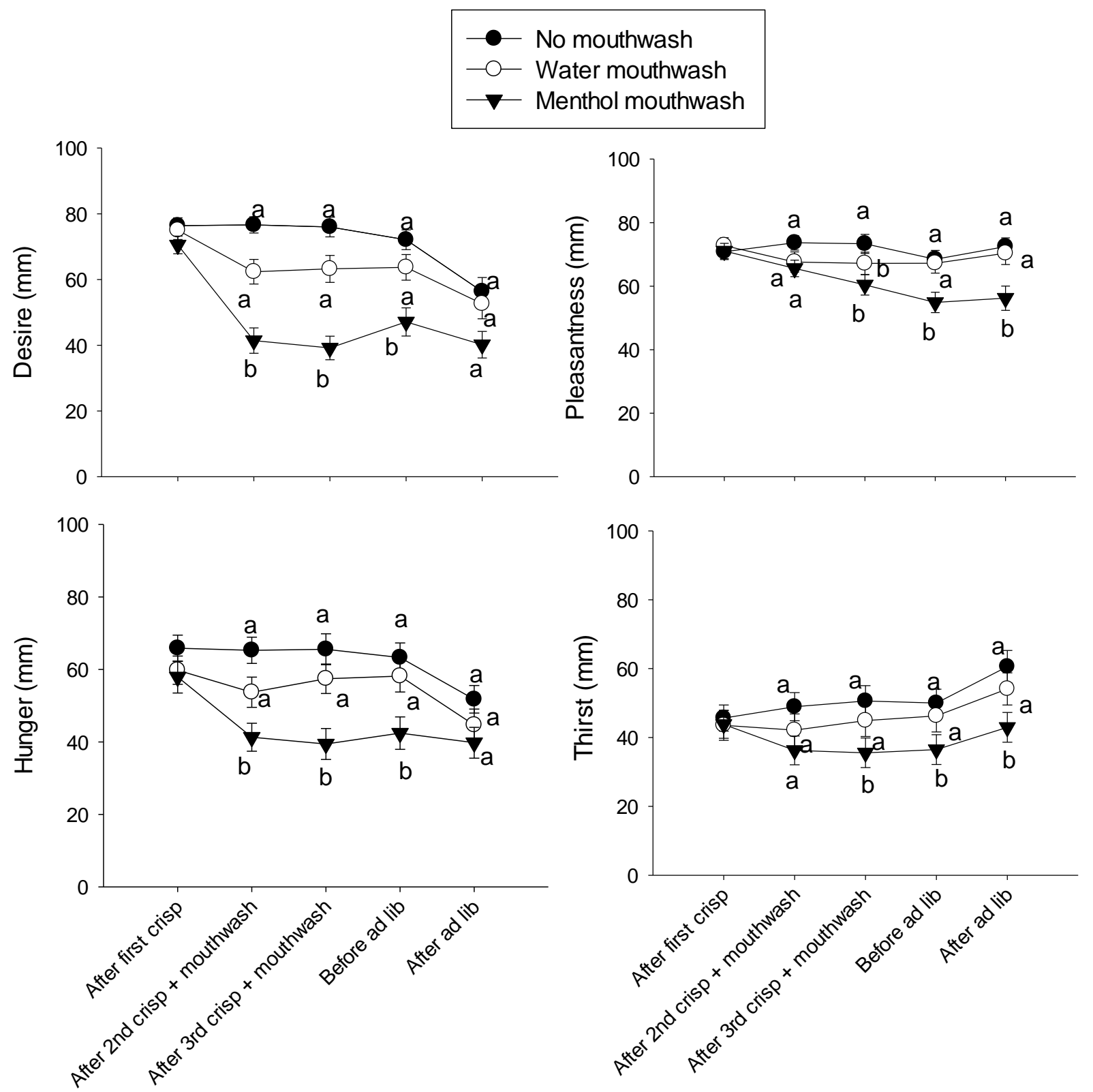

Fig. 4: Visual analogue scales for desire, hunger, pleasantness and thirst $(n=36)($ mean $\pm S E)$. Scales were anchored with the markers of $0=$ 'not at all' and $100=$ 'very' with the questions: How strong is your desire to eat more crisps right now?, How hungry do you feel right now? Please rate the pleasantness of the crisps you just swallowed, How thirsty do you feel right now?. Different vertical letters at each interval indicate significant post hoc differences according to a pairwise Freidman test using a Bonferroni correction $(\mathrm{p}<0.05, \alpha=0.05 / 3=$ 0.016). 


\subsubsection{Crisp intake for ad libitum task}

The quantity of crisps consumed for all mouthwash conditions at each 3 minute time interval throughout the 12 minute task is shown in Fig. 5. At the end of the ad libitum exercise (12 minutes), a significant difference in weight consumed was found between the three conditions according to the Friedmans test $\chi^{2}(2, \mathrm{~N}=36)=12.623, \mathrm{p}<0.005$. Post hoc results, shown in Fig. 5, found the total weight consumed during the menthol mouthwash treatment was significantly less than during the no mouthwash treatment and during the water mouthwash treatment. Similar trends were observed at all 3 minute intervals (where the mouthwash treatments or no mouthwash (pause) was applied). 


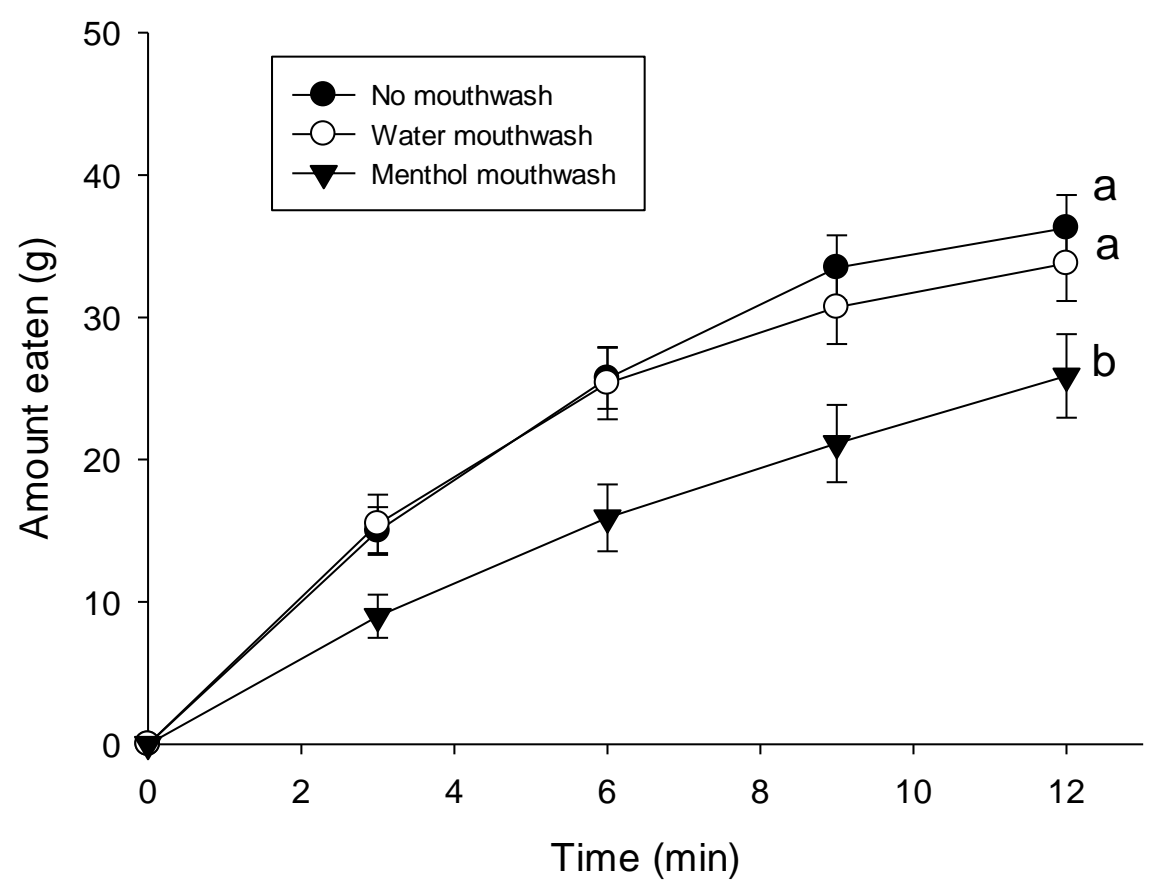

Fig. 5: Amount of crisps eaten during the ad libitum task over a 12 minute period, where mouthwash interventions were conducted every three minutes (at $t=0 \min , t=3 \min , t=6 \min$, and $t=9 \mathrm{~min}$ ), and weight consumed was also measured every 3 minutes (mean $\pm \mathrm{SE}$ ).

Different vertical letters at 12 minutes indicate significant post hoc differences at the end of the ad libitum session, according to a pairwise Freidman test using a Bonferroni correction ( $\mathrm{p}$ $<0.05, \alpha=0.05 / 3=0.016$ ). 


\section{Discussion}

This study has made a number of novel findings. Firstly, it has shown that a water mouthwash reduces the perception of aftertaste attributes with potato crisps (measured for the first time using a TDS), but that this change in aftertaste does not influence appetite according to VAS scales and ad libitum intake. Secondly, it has shown that a menthol mouthwash generates contrasting aftertaste attributes (minty, cooling, tingly), and this change in aftertaste induces a reduction in appetite according to VAS scales and ad libitum intake. Finally, it has shown that the TDS technique can be used with the intervention of a mouthwash in the middle of a recording sequence, where the sensory experience both before and after the mouthwash (aftertaste) are recorded. Detailed discussion is provided below.

\subsection{Sensory study}

The experimental design was successful in modifying the aftertaste of potato crisps according to the TDS sensory methodology. The application of a water mouthwash reduced the dominant selection of savoury, salty and fatty mouth coating aftertaste attributes, while the menthol mouthwash generated minty, cooling and tingly aftertaste attributes (Fig. 3). Without any mouthwash, the aftertaste sensations of savoury, salty, and fatty mouthcoating persisted at significant dominant rates right up to the end of the 90 s period.

The application of a water rinse has been previously shown to reduce aftertastes with different foods/ingredients (Nasrawi \& Pangborn 1990; Allison et al., 1999; Lucak \& Delwiche, 2009), however prior to this study it had never been investigated using the TDS method. Ng et al. (2012) showed perception of attributes from blackcurrant squashes persisted for up to 60s with TDS (no mouthwash). In the case of this study the water mouthwash is likely to have removed most of the residual food bolus and mouthcoating of the crisp that remains after swallowing. However, it appears some residue/mouthcoating still remains, given that savoury and salty attributes are still selected at rates around $10-15 \%$ (above the chance level) for most of the time period after the water mouthwash. In terms of the menthol mouthwash, its significantly stronger taste profile appears to have suppressed any savoury and salty tastes remaining from a residue/ mouthcoating, and its $1 \% \mathrm{v} / \mathrm{v}$ ethanol content may also support the removal of hydrophobic (fat/oil) components from the mouthcoating.

The sensory experience prior to swallowing (ie during mastication) did not appear to be influenced by changing the type of mouthwash treatment. No menthol terms (minty, cooling, tingly) were selected by TDS subjects during the mastication phase of the menthol session, showing that a sensory carry over from menthol did not influence sensory perception during the VAS scales testing from the 2nd crisp to the 3rd crisp. Menthol has a long time course (Gwartney \& Heymann, 1995), however the palate 
cleansing procedure between mouthwashes appeared effective in removing any sensory carry over effect for a subsequent crisp.

The evolution in sensory terms leading up to swallowing reflects results observed in previous TDS studies with peanuts (Hutchings et al., 2014b), and is typical of what would be expected as the crisps are fractured and reduced in size by the teeth, flavours released, and the food bolus formed. Potato crisps are comprised of cellular material, which is $70 \%$ porous, and will fracture, accompanied by a loud sound, with only a small application of force by the teeth (Rojo and Vincent, 2008).

TDS appeared to cope well with a mouthwash intervention mid sequence (after swallowing), as a clear progression in the selection of dominant attributes can be seen prior to the mouthwash $(0-23 \mathrm{~s})$ and after the mouthwash (46-90s) (Fig. 3). There was a large shift in the profile of dominant attributes following both mouthwash treatments, which indicates subjects responded reasonably quickly to the change in sensory experience after each mouthwash. It also indicates subjects responded well to the instruction to expectorate the mouthwash into a container (at 46s), and then to the instruction to immediately recommence the TDS task. However, for both mouthwash treatments a lag can be seen from approximately $46-55 \mathrm{~s}$, as the subjects took some time to select new attributes (thereby unselecting attributes which were dominant at $23 \mathrm{~s}$ when the mouthwash period began). (Subjects were instructed not to make any attribute selections during the mouthwash period - logistically this was too difficult as their attention was needed to control the mouthwashes).

\subsection{Appetite study}

The aftertaste modification via the menthol mouthwash sufficiently influenced aftertaste and reduced pleasantness and desire for potato crisps (as well as hunger and thirst), when compared to the no mouthwash and water mouthwash treatments. This modification in aftertaste may be one explanation for the changes in consumption observed during the ad libitum task. The menthol mouthwash also modified appetite so that subjects entered the ad libitum task with reduced desire to eat, pleasantness, thirst and hunger ratings. Although the importance of orosensory reward pathways in the consumption of pleasurable foods is well known (Mela, 2006; Finlayson \& Dalton, 2012; Yeomans, 2000), what is novel is the measurable drop in desire, hunger, and consumption (in a laboratory setting) when manipulating only the aftertaste. The crisps served did not change between treatments, however the incongruent presence of minty, cooling, tingly sensations in the mouth after crisp consumption may have reduced desire for more savoury, salty crisps.

Understanding the reduction in desire to eat and ad libitum intake of the crisps due to the menthol mouthwash is complex, and could be attributed to both psychological and physiological factors. From 
a psychological standpoint, it has been previously reported that food odours decrease the appetite of mis-matched foods (Ramaekers et al., 2014 b\&c) and therefore the introduction of incongruent (mismatching) attributes of minty, cooling and tingly disrupted the oro-sensory experience to cause measurable reductions in the VAS scales and reduced intake of the crisps. The sensory attributes of menthol give the feeling of a 'clean' mouth, and menthol is not typically consumed with crisps. Furthermore menthol sensations are often associated with products linked to the cessation of eating; such as toothpaste, mouthwash, and chewing gum.

Cephalic phase responses (sensory signals inducing physiological responses for the digestion of food (Smeets, Ekner, \& de Graaf, 2010)) to menthol may also be important. The incongruent and 'non-food' sensory profile of menthol may induce a suppression in the body's natural cephalic response to ingest crisps. Modified Sham Feeding studies show that sensory perception without ingestion can contribute to physiological changes in appetite (Smeets \& Westerterp-Plantenga, 2006). Menthol also stimulates the TRPM8 thermoreceptor (Macpherson et al., 2006) to generate the sensation of cooling, and in some studies has this been shown to influence thirst and arousal (Eccles, 2000), and breathing behaviour (Eccles, 2003; Mundel \& Jones, 2009). Ethanol, used in this study as a solvent at $1 \% \mathrm{v} / \mathrm{v}$ to dissolve the menthol to produce the mouthwash is very unlikely to cause any sensory or physiological effect given its low concentration (it can induce the sensation of heat but only at much higher concentrations (Nolden \& Hayes, 2015).

While the water mouthwash was successful in dramatically lowering savoury and salty attributes after crisp consumption, there were only small, non-significant reductions in the VAS scales (desire, hunger, and pleasantness) and ad libitum intake after the water mouthwash. Data in the literature suggests a possible role for residual odours and tastes left in the mouth to prime or increase food intake (Sorensen et al., 2003; Prescott, 2012). However, in the present study, this result was not observed. This data may suggest that after swallow residual flavours do not act as a strong trigger for further consumption; however a study with larger numbers of subjects may have shown a significant effect.

The results of this study conflict somewhat with the body of literature on SSS. Why does the menthol mouthwash reduce food intake rather than increasing it? And why does the water mouthwash have no influence on food intake rather than increasing it? The phenomenon of SSS occurs when a particular food is eaten, its pleasantness gradually decreases, while the pleasantness of alternative foods remains unchanged. Subsequent ad libitum intake of that food is typically less than alternative foods (Rolls et al., 1981). Even the sensory experience of chewing or olfactory sensations that would be experienced over the time course of a meal for a given food, without actual food intake, has been shown to reduce pleasantness of that particular food, but not to influence the pleasantness of other foods (Rolls \& Rolls, 1997). 
The authors propose that, in the case of the menthol, SSS was not the main driver to influence appetite and food intake, but instead the incongruent 'aftertaste' /'mouthcoating' comprised of minty/cooling sensations was a stronger driver, making the savoury/salty crisps seem more unpleasant to the participant. Again this may be due to the 'non-food' nature of menthol, its sensory profile typically associated with non-food items. SSS studies showing increased appetite and food intake through sensory variety do so with food products that are non-mismatched: ie they are typically consumed together (Rolls et al., 1981; Guinard \& Brun, 1998).

Understanding any role of SSS in the case of the water mouthwash is perhaps even more difficult than with the menthol, given that no significant effect was observed. It is possible that a reduction in priming achieved by the water mouthwash was a stronger driver reducing appetite than any increase in appetite by removing aftertaste and reducing SSS. It is also possible the magnitude of reduced sensations from the water mouthwash was not sufficient to induce any increase in appetite according to SSS theory (most of the sensory stimulation inducing satiety is likely to be delivered during mastication of the crisp).

\subsection{Ecological relevance of this study}

This study offers two key potential strategies to reduce the intake of high fat savoury products, such as potato crisps:

(1) Practical intervention strategies using common food items that have a sensory profile dominated by menthol or mint. For example, if a consumer finds themselves snacking on too many crisps during a given eating occasion, one potential strategy could be intervening by having a peppermint tea, menthol flavoured chewing gum, or brushing their teeth, to slow down or stop snacking. Any product that offers the incongruent minty, cooling, tingly sensations of menthol may be effective.

(2) Controlled release strategies for high fat savoury products. In years to come it may be possible to encapsulate a tastant like menthol within a food structure, prevent it from being perceived over the short term, thus having no influence on initial palatability of a product, but by attachment to the oral mucosa and eventual tastant release in the mouth, snacking could be dictated to cease (eg after 10 minutes). 


\section{Conclusion}

Sensory analysis, conducted using the Temporal Dominance of Sensations methodology (TDS), showed that a water mouthwash reduced aftertaste attributes such as savoury, salty, and fatty mouthcoating, in comparison with a no mouthwash control. A menthol mouthwash increased aftertaste attributes of cooling, minty, and tingly in comparison with a no mouthwash control. The water mouthwash did not influence desire and liking of the crisps, or hunger and thirst, according to the VAS scales. The water mouthwash did not influence the ad libitum intake of the crisps over a 12 minute period. The menthol mouthwash significantly reduced desire and liking of the crisps, as well as hunger and thirst, according to the VAS scales. The menthol mouthwash also significantly reduced ad libitum crisp intake by $29 \%$ over the 12 minute period. Results suggest the intake of high fat savoury products could be reduced by consumers intervening with the products containing these incongruent (minty, cooling, tingly) sensations during snacking. Further work is required to establish underlying mechanisms and the effects of longer term interventions manipulating aftertaste on appetite control and food intake.

\section{Acknowledgements}

This study was funded by the University College Dublin Seed Funding Scheme. The authors would like to thank Emer Garvey for her assistance in the sensory study. 


\section{References}

Allison, A.-M. A., Iv, E. C., Milliken, G. A., \& Chambers, D. H. (1999). Effects of interstimulus rinsing and time on measurements of capsaicin heat in tomato salsa. Journal of Sensory Studies, 14(4), 401-414.

Duizer, L. M., Bloom, K., \& Findlay, C. J. (1997). Dual-attribute time-intensity sensory evaluation: A new method for temporal measurement of sensory perceptions. Food Quality and Preference, 8(4), 261-269.

Eccles, R. (1994). Menthol and Related Cooling Compounds. Journal of Pharmacy and Pharmacology, 46(8), 618-630.

Eccles, R. (2000). Role of cold receptors and menthol in thirst, the drive to breathe and arousal. Appetite, 34(1), 29-35.

Eccles, R. (2003). Menthol: Effects on nasal sensation of airflow and the drive to breathe. Current Allergy and Asthma Reports, 3(3), 210-214.

Finlayson, G. and Dalton, M. (2012). Hedoncis of food consumption: are food 'liking' and 'wanting' viable targets for appetite control in the obese? Current obesity reports. 1(1), 42-49.

Forde, C. G., van Kuijk, N., Thaler, T., de Graaf, C., \& Martin, N. (2013a). Oral processing characteristics of solid savoury meal components, and relationship with food composition, sensory attributes and expected satiation. Appetite, 60, 208-219.

Forde, C. G., van Kuijk, N., Thaler, T., de Graaf, C., \& Martin, N. (2013b). Texture and savoury taste influences on food intake in a realistic hot lunch time meal. Appetite, 60, 180-186.

Forslund, H.B., Torgerson, J. S., Sjostrom, L., \& Lindroos, A. K. (2005). Snacking frequency in relation to energy intake and food choices in obese men and women compared to a reference population. International Journal of Obesity, 29(6), 711-719.

Gaillet, M., Sulmont-Rossé, C., Issanchou, S., Chabanet, C., \& Chambaron, S. (2013). Priming effects of an olfactory food cue on subsequent food-related behaviour. Food Quality and Preference, 30(2), 274-281.

Gaillet-Torrent, M., Sulmont-Rossé, C., Issanchou, S., Chabanet, C., \& Chambaron, S. (2014). Impact of a non-attentively perceived odour on subsequent food choices. Appetite, 76, 17-22.

Green, B. G. (1988). Spatial and temporal factors in the perception of ethanol irritation on the tongue. Perception \& Psychophysics, 44(2), 108-116.

Green, B. G., \& Hayes, J. E. (2003). Capsaicin as a probe of the relationship between bitter taste and chemesthesis. Physiology \& Behavior, 79(4-5), 811-821.

Guinard, J.-X., \& Brun, P. (1998). Sensory-specific Satiety: Comparison of Taste and Texture Effects. Appetite, 31(2), 141-157.

Gwartney, E., \& Heymann, H. (1995). The temporal perception of menthol. Journal of Sensory Studies, 10(4), 393-400. 
Hutchings, S. C., Foster, K. D., Grigor, J. M. V., Bronlund, J. E., \& Morgenstern, M. P. (2014b). Temporal dominance of sensations: A comparison between younger and older subjects for the perception of food texture. Food Quality and Preference, 31(0), 106-115.

Hutchings, S. C., Foster, K. D., Hedderley, D. I., \& Morgenstern, M. P. (2014a). Differences between Age Groups in the Use of the Temporal Dominance of Sensations Technique across a Range of Food Textures. Journal of Texture Studies, 45(3), 206-219.

Labbe, D., Schlich, P., Pineau, N., Gilbert, F., \& Martin, N. (2009). Temporal dominance of sensations and sensory profiling: A comparative study. Food Quality and Preference, 20(3), 216-221.

Lucak, C. L., \& Delwiche, J. F. (2009). Efficacy of Various Palate Cleansers with Representative Foods. Chemosensory Perception, 2(1), 32-39.

Macpherson, L. J., Hwang, S. W., Miyamoto, T., Dubin, A. E., Patapoutian, A., \& Story, G. M. (2006). More than cool: Promiscuous relationships of menthol and other sensory compounds. Molecular and Cellular Neuroscience, 32(4), 335-343.

McCrickerd, K., \& Forde, C. G. (2015). Sensory influences on food intake control: moving beyond palatability. Obesity Reviews, 17(1), 18-29.

Mela, D.J. (2006). Eating for pleasure or just wanting to eat ? Reconsidering sensory hedonic responses as a driver of obesity. Appetite, 47, 10-17.

Mündel, T., \& Jones, D. A. (2009). The effects of swilling an 1(-)-menthol solution during exercise in the heat. European Journal of Applied Physiology, 109(1), 59-65.

Nasrawi, C. W., \& Pangborn, R. M. (1990). Temporal effectiveness of mouth-rinsing on capsaicin mouth-burn. Physiology \& Behavior, 47(4), 617-623.

Ng, M., Lawlor, J. B., Chandra, S., Chaya, C., Hewson, L., \& Hort, J. (2012). Using quantitative descriptive analysis and temporal dominance of sensations analysis as complementary methods for profiling commercial blackcurrant squashes. Food Quality and Preference, 25(2), 121-134.

Nolden, A. A., \& Hayes, J. E. (2015). Perceptual Qualities of Ethanol Depend on Concentration, and Variation in These Percepts Associates with Drinking Frequency. Chemosensory Perception, 8,149157.

Pineau, N., \& Schlich, P. (2014). Temporal Dominance of Sensations (TDS) as a sensory profile technique. In J. Delarue, B. Lawlor, \& M. Rogeaux. (Eds.), Rapid Sensory Profile Techniques and Related Methods: Applications in New Product Development and Consumer Research (pp. 269-304). Cambridge: Woodhead Publishing.

Pineau, N., Schlich, P., Cordelle, S., Mathonnière, C., Issanchou, S., Imbert, A., Rogeaux, M., Etiévant, P., \& Köster, E. (2009). Temporal Dominance of Sensations: Construction of the TDS curves and comparison with time-intensity. Food Quality and Preference, 20(6), 450-455.

Prescott, J. (2012). Chemosensory learning and flavour: perception, preference and intake. Physiology and Behaviour, 107(4), 553-559. 
Ramaekers, M. G., Boesveldt, S., Gort, G., Lakemond, C. M. M., van Boekel, M. A. J. S., \& Luning, P. A. (2014c). Sensory-Specific Appetite Is Affected by Actively Smelled Food Odors and Remains Stable Over Time in Normal-Weight Women. The Journal of Nutrition, 144(8), 1314-1319.

Ramaekers, M. G., Boesveldt, S., Lakemond, C. M. M., van Boekel, M. A. J. S., \& Luning, P. A. (2014b). Odors: appetizing or satiating? Development of appetite during odor exposure over time. International Journal of Obesity, 38(5), 650-656.

Ramaekers, M. G., Luning, P. A., Ruijschop, R. M. A. J., Lakemond, C. M. M., Bult, J. H. F., Gort, G., \& van Boekel, M. A. J. S. (2014a). Aroma exposure time and aroma concentration in relation to satiation. British Journal of Nutrition, 111(03), 554-562.

Rogers, P. J., \& Hardman, C. A. (2015). Food reward. What it is and how to measure it. Appetite, 90, $1-15$.

Rojo, F.J. and Vincent, J.F.V., 2008. Fracture properties of potato crisps. International Journal of Food Science \& Technology, 43(4), pp.752-760.

Rolls, B. J., Rolls, E. T., Rowe, E. A., \& Sweeney, K. (1981). Sensory specific satiety in man. Physiology \& Behavior, 27(1), 137-142.

Rolls, E. T., \& Rolls, J. H. (1997). Olfactory Sensory-Specific Satiety in Humans. Physiology \& Behavior, 61(3), 461-473.

Ruijschopa, R. M. A. J., Boelrijk., A. E. M., de Ru, J.A., de Graaf C., and Westerterp-Plantenga, M S. (2008). Effects of retro-nasal aroma release on satiation. British Journal of Nutrition, 99(5), 1140-1148.

Smeets, A. J., \& Westerterp-Plantenga, M. S. (2006). Satiety and substrate mobilization after oral fat stimulation. British Journal of Nutrition, 95(04), 795-801

Smeets, P. A., Erkner, A., \& de Graaf, C. (2010). Cephalic phase responses and appetite. Nutrition Reviews, 68(11), 643-655.

Sørensen, L.B., Møller, P., Flint, A., Martens, M. and Raben, A.(2003) Effect of sensory perception of foods on appetite and food intake: a review of studies on humans. International Journal of Obesity, $27,1152-1166$.

Weijzen, P. L. G., Smeets, P. A. M., \& de Graaf, C. (2009). Sip size of orangeade: effects on intake and sensory-specific satiation. British Journal of Nutrition, 102(07), 1091-1097.

Yeomans, M.R. (2000). Rating changes over the course of meals: what do they tell us about motivation to eat? Neurosicience and Biobehavioural Reviews, 24, 249-259. 